\title{
A comparison between two different automated total 25-hydroxyvitamin D immunoassay methods using liquid chromatography-tandem mass spectrometry
}

\author{
Fatma Emel Kocak ${ }^{* 1}$, Bahadir Ozturk², Ozben Ozden Isiklar ${ }^{3}$ Ozlem Genc' ${ }^{4}$, Ali Unlu², Irfan Altuntas ${ }^{1}$ \\ 1Dumlupinar University, Faculty of Medicine, Department of Medical Biochemistry, Kutahya, Turkey \\ ${ }^{2}$ Selcuk University, Faculty of Medicine, Department of Medical Biochemistry, Konya, Turkey \\ ${ }^{3}$ Dumlupinar University, Evliya Celebi Education and Research Hospital, Department of Medical Biochemistry, Kutahya, Turkey \\ ${ }^{4}$ Dumlupinar University, Faculty of Medicine, Department of Medical Microbiology, Kutahya, Turkey \\ *Corresponding author: dremelk@hotmail.com
}

\begin{abstract}
Introduction: Total 25-hydroxyvitamin D [25(OH)D] is the most reliable indicator of vitamin D status. In this study, we compared two automated immunoassay methods, the Abbott Architect 25-OH Vitamin D assay and the Roche Cobas Vitamin D total assay, with the liquid chromatographytandem mass spectrometry (LC-MS/MS).

Materials and methods: One hundred venous blood samples were randomly selected from routine vitamin $D$ tests. Two of the serum aliquots were analyzed at the Abbott Architect i2000 and the Roche Cobas 6000's module e601 in our laboratory within the same day. The other serum aliquots were analyzed at the LC-MS/MS in different laboratory. Passing-Bablok regression analysis and Bland-Altman plot were used to compare methods. Inter-rater agreement was analyzed using kappa (K) analysis.

Results: The Roche assay showed acceptable agreement with the LC-MS/MS based on Passing-Bablok analysis (intercept: $-5.23 \mathrm{nmol} / \mathrm{L}, 95 \% \mathrm{Cl}$ : -8.73 to 0.19 ; slope: $0.97,95 \%$ Cl: 0.77 to 1.15 ). The Abbott assay showed proportional (slope: $0.77,95 \%$ Cl: 0.67 to 0.85 ) and constant differences (intercept: $17.08 \mathrm{nmol} / \mathrm{L} ; 95 \% \mathrm{Cl}: 12.98$ to 21.39 ). A mean bias of $15.1 \%$ was observed for the Abbott and a mean bias of $-14.1 \%$ was observed for the Roche based on the Bland-Altman plots. We found strong to nearly perfect agreement in vitamin D status between the immunoassays and LC-MS/ MS. (к: 0.83 for Abbott, к: 0.93 for Roche) using kappa analysis.

Conclusion: Both immunoassays demonstrated acceptable performance, but the Roche Cobas assay demonstrated better performance than the Abbott Architect in the studied samples.
\end{abstract}

Key words: 25-Hydroxyvitamin D; chromatography; immunoassay; methods; tandem mass spectrometry

\section{Introduction}

Vitamin D, also known as the "sunshine vitamin," is a lipid-soluble nutrient that is obtained from dietary sources as ergocalciferol (D2) or cholecalciferol (D3) (1). Although vitamin D is classically known for its role in bone metabolism, it plays an important role in the body beyond its functions in the musculoskeletal system (2). Previous studies have reported that vitamin $D$ is not only required for bone health, but also plays a role in autoimmune diseases, obesity, type 2 diabetes mellitus, meta- bolic syndrome, cardiovascular diseases, and certain types of cancer. These reports have led to an increase in requests for vitamin $D$ tests by clinicians in recent years (3-5).

25-Hydroxyvitamin D [25(OH)D] (calcidiol) has a half-life of several weeks; therefore, the most accurate method of determining vitamin $D$ status is to measure 25(OH)D. Serum 25(OH)D concentration represents endogenous vitamin $\mathrm{D}$ synthesis in the skin as well as dietary intake. Measurement of 
1,25-dihydroxyvitamin $\mathrm{D}\left[1,25(\mathrm{OH})_{2} \mathrm{D}\right]$ (calcitriol) is not a good indicator of the vitamin $D$ status, as it has a very short half-life of approximately $4 \mathrm{~h}$, and its blood levels are closely regulated by the serum levels of parathyroid hormone, calcium, and phosphate. Calcitriol concentration also does not reflect the vitamin D reserves, as levels are frequently elevated in individuals with hypovitaminosis D because of secondary hyperparathyroidism. Therefore, total $25(\mathrm{OH}) \mathrm{D}$ is the most reliable indicator of vitamin D status $(6,7)$.

Although the use of vitamin D testing has recently increased substantially, there is little consensus on which assay should be used to measure its concentration, and there are serious concerns regarding the reliability of its measurement (7). To correctly assess vitamin $D$ status, a method for reliably measuring $25(\mathrm{OH}) \mathrm{D}$ is needed. Several specifications should be considered when selecting a vitamin $\mathrm{D}$ assay, including total $25(\mathrm{OH}) \mathrm{D}$ measurement (the sum of $25(\mathrm{OH}) \mathrm{D}_{2}$ and $25(\mathrm{OH}) \mathrm{D}_{3}$ ), accuracy, reproducibility, turn-around time, inter-assay comparability, and cost- effectiveness (8). Until recently, no generally accepted reference method for 25(OH)D measurement was available. In 2010, Tai et al. developed isotope dilution liquid chromatography-tandem mass spectrometry (LC-MS/MS) as a candidate reference method, which in 2011 was recognized as a reference method by the Joint Committee for Traceability in Laboratory Medicine (9). Isotope dilution LC-MS/MS is currently considered the reference method for $25(\mathrm{OH}) \mathrm{D}$ measurement, as it can simultaneously quantitate $25(\mathrm{OH})$ $\mathrm{D}_{2}$ and $25(\mathrm{OH}) \mathrm{D}_{3}$; these values are summed to determine total 25(OH)D (10). Recently, some manufacturers have developed automated immunoassays for 25(OH)D measurement, including Siemens, IDS, Abbott, Roche, and DiaSorin. Except for the Roche assay, all current automated tests use a similar method design. Sample pretreatment dissociates $25(\mathrm{OH}) \mathrm{D}$ from vitamin $\mathrm{D}$ binding protein (VDBP), and 25(OH)D competes with exogenous labeled 25(OH)D for binding to assay specific antibodies. The Roche assay is a competitive proteinbinding assay and uses exogenous recombinant human VDBP to capture $25(\mathrm{OH}) \mathrm{D}$ in the patient sample (11).
Because 25(OH)D is highly lipophilic and has strong binding affinity for VDBP, it is not easy or simple to measure its concentration in patient sera. There is a great deal of variety among $25(\mathrm{OH})$ $\mathrm{D}$ assays because of the different techniques employed for separating 25(OH)D from its binding protein, as well as its detection and measurement (12). Although LC-MS/MS is considered the reference method for measuring $25(\mathrm{OH}) \mathrm{D}$ concentrations, the instrument is very expensive, unavailable in most clinical laboratories, and its turnaround time is relatively longer than that of immunoassays. Therefore, automated and high-throughput immunoassays may be a good alternative for clinical laboratories. However, there is no strong agreement between the current immunoassay methods because of intermethod variability due to the different standardizations used $(12,13)$. In cooperation with the National Institutes of Health's Office of Dietary Supplements, the National Institute of Standards and Technology (NIST) developed standard reference materials (SRMs) in order to improve accuracy and to enable worldwide standardization for 25(OH)D measurement. The SRM 2972 contains defined amounts of $25(\mathrm{OH}) \mathrm{D}_{2}$ and $25(\mathrm{OH}) \mathrm{D}_{3}$ dissolved in ethanol. This material is used for direct calibration of LC-MS/MS reference measurement procedures. While this material can be used with chromatographic methods as calibrators, the antigen-antibody reaction prevents direct calibration of immunological methods by using this calibration solution. Therefore, serumbased materials are needed for calibration of immunological methods. The NIST developed serum-based reference material, SRM 972, which consists of four pools of human serum with analyte values for $25(\mathrm{OH}) \mathrm{D}_{2}, 25(\mathrm{OH}) \mathrm{D}_{3}$, and 3-epi$25(\mathrm{OH}) \mathrm{D}_{3}$. Despite these standardization efforts, unresolved discrepancies and unacceptable biases persist between immunoassays compared to the reference methods. Therefore, further standardization studies for 25(OH)D measurements should be conducted to obtain reliable results and to correctly define the vitamin $D$ status in patients (14).

Vitamin D testing orders have increased by 20 -fold in the past 3 years in our laboratory, with nearly $75 \%$ of results indicating vitamin D deficiency or 
insufficiency. Therefore, in this study we compared two automated immunoassay methods, the Abbott Architect 25-OH Vitamin D assay and the Roche Cobas Vitamin D total assay, while referencing the LC-MS/MS method to verify our routine 25(OH)D measurements. In this study, methods were compared by considering recommendations of the Clinical and Laboratory Standards Institute (CLSI) Evaluation Protocol 9 (EP09-A3) specifications (15). This document provides guidance for designing an experiment and selecting methods for quantifying systematic measurement error (bias or difference) between measurement procedures based on comparison of patient samples. Given the the increase in use of vitamin D testing day by day, findings of this study would provide substantial data about comparability of commonly used two immunoassay methods with LC-MS/ MS method for laboratory professionals.

\section{Materials and methods}

\section{Study design}

This method comparison study was carried out at the Evliya Celebi Research and Education Hospital of Dumlupinar University and the Medical Faculty Hospital of Selcuk University, Turkey in November 2014. The study was carried out in accordance with the Declaration of Helsinki. One hundred venous blood samples from 70 adult females and 30 adult males were randomly selected from our routine vitamin $D$ test requests. The total number of daily $25(\mathrm{OH}) \mathrm{D}$ test requests is $110-120$ in our hospital.

\section{Collection of samples and measurement of serum total 25(OH)D}

After an overnight fasting, venous blood samples were collected into an evacuated serum separator clot activator tube (Vacuette ${ }^{\circledR}$ Z Serum Sep Clot Activator, GreinerBio-One, Kremsmunster, Austria) between 9 a.m. and 10 a.m. Blood samples were centrifuged at $1500 \times \mathrm{g}$ for $10 \mathrm{~min}$ within $1 \mathrm{~h}$ of collection. Hemolytic, lipemic, and icteric serum samples and samples collected in inadequate test tubes or samples with insufficient volume were excluded from the study. The serum samples were aliquoted into three separate polystyrene tubes. Two aliquots were immediately processed on the following platforms: Abbott Architect i2000 (Abbott Laboratories, Wiesbaden, Germany) by chemiluminescent microparticle immunoassay using Abbott Architect 25-OH Vitamin D assay reagent (Abbott Laboratories, Wiesbaden, Germany) and Roche Cobas 6000's module e601 (Roche Diagnostics $\mathrm{GmbH}$, Mannheim, Germany) by electrochemiluminescence immunoassay using Roche Cobas Vitamin D total assay reagent (Roche Diagnostics $\mathrm{GmbH}$, Mannheim, Germany). Samples were processed in a single batch in duplicate on each analyzer according to the manufacturer's instructions. Calibration curves were constructed using calibrators provided in the kits. The third aliquot was stored at $-20^{\circ} \mathrm{C}$. After 1 week of storage, collected aliquots were transported to the Medical Faculty Hospital of Selcuk University on dry ice for 25(OH)D analysis by LC-MS/MS. Before analysis, frozen samples were thawed and single processing was conducted. The LC-MS/MS assay method was adapted from a method described by Sahillioglu et al. (16). MS detection was carried out with an AB Sciex API 3200 triple quadrupole liquid tandem mass spectrometer (AB Sciex Instruments, Biopolis, Singapore) using atmospheric pressure chemical ionisation as the ionization source. Multiple reaction monitoring positive ion mode was used to detect 25-hydroxy metabolites of vitamin $\mathrm{D}$ and deuterium-labeled internal standard $[2 \mathrm{H} 6-25(\mathrm{OH})$ $D_{3}$ ] (DLM-9116, Cambridge Isotope Laboratories, Inc., Andover, MA, USA). Chromatographic separation of $25(\mathrm{OH}) \mathrm{D}_{3}$ was carried out using a chromatography unit (Shimadzu Prominence SIL LC-20, Shimadzu Corporation, Kyoto, Japan) and a C18 reverse phase column (Phenomenex, Luna Analytical Column, Torrance, CA, USA). The samples were prepared using a protein precipitation protocol and the analyte was extracted by protein crash with acetonitrile. Quantitation was performed by linear regression of peak area ratios of $25(\mathrm{OH}) \mathrm{D}_{3} /$ $2 \mathrm{H} 6-25(\mathrm{OH}) \mathrm{D}_{3}$ against the calibrator concentrations (IC3402ka CAL, ImmuChrom GmbH, Heppenheim, Germany). Measurement ranges of the assays were $20.0-400.0 \mathrm{nmol} / \mathrm{L}$ for the Abbott Architect $25-\mathrm{OH}$ Vitamin D assay, 7.5-175.0 nmol/L for 
the Roche Cobas Vitamin D total assay, and 10.0$2500.0 \mathrm{nmol} / \mathrm{L}$ for the LC-MS/MS assay. Internal quality control samples were included in the assay run and intra-assay coefficients of variation (CVs) values were obtained from the measurements. For Architect i2000 analysis, intra-assay CVs were 4.2\%, $2.7 \%$, and $2.2 \%$, with target values of $47.5 \mathrm{nmol} / \mathrm{L}$, $95.0 \mathrm{nmol} / \mathrm{L}$, and $195.0 \mathrm{nmol} / \mathrm{L}$ (Architect $25-\mathrm{OH}$ Vitamin D Control L, M, and H, Abbott Laboratories, Wiesbaden, Germany). For Cobas e601analysis, intra-assay CVs were $4.9 \%$ and $3.1 \%$, with target values of $50.5 \mathrm{nmol} / \mathrm{L}$, and $99.0 \mathrm{nmol} / \mathrm{L}$ (PreciControl Varia 1 and 2, Roche Diagnostics GmbH, Mannheim, Germany). For LC-MS/MS analysis, intra-assay CVs were $3.8 \%$ and $2.3 \%$, with target values of $57.1 \mathrm{nmol} / \mathrm{L}$ and $127.0 \mathrm{nmol} / \mathrm{L}$ (IC3402ko CTRL 1 and 2, ImmuChrom GmbH, Heppenheim, Germany).

\section{Statistical analysis}

The $25(\mathrm{OH}) \mathrm{D}$ results obtained by LC-MS/MS were used as the reference for method comparison studies. Results reading below or above the lower or upper limit of measurement ranges of the immunoassay methods were omitted from statistical evaluation. Concentrations of 25(OH)D were given in $\mathrm{nmol} / \mathrm{L}$ International System of Units (SI). All data sets were tested for normality using Kolmogorov-Smirnov test. Passing-Bablok regression analysis was used to assess constant and proportional biases between methods, including the Cusum test for linearity. A P value $<0.05$ indicates a significant deviation from linearity. For significant agreement, the $95 \%$ confidence interval $(\mathrm{Cl})$ of the intercept should contain the zero, while the $95 \% \mathrm{Cl}$ of the slope should contain 1 (17). A Bland-Altman plot was used to assess differences and biases between methods. Bland and Altman recommend plotting differences against the average of the methods rather than against that of the reference method, while CLSI recommends plotting differences against the reference method $(15,18)$. Therefore, differences between values from comparative immunoassays and the reference method against the reference method value were displayed in the difference plots according to CLSI recommendations. The differences expressed as a percentage of the reference method value were plotted to illustrate whether the difference between the measurements made using the two methods was related to the magnitude of the measurement. Inter-rater agreement in assessment of vitamin $D$ status between assays was analyzed using kappa (K) analysis (19). A kappa value of 1.0 indicates perfect agreement, $>0.90$ indicates almost perfect agreement; 0.80-0.90 indicates strong agreement, 0.60-0.79 indicates moderate agreement, 0.40-0.59 indicates weak agreement, $0.21-0.39$ indicates minimal agreement, and $<0.20$ indicates no agreement between methods. Statistical analysis was performed using MedCalc software (version 9.2.0.1, MedCalc Software, Ostend, Belgium).

\section{Results}

25(OH)D results obtained by LC-MS/MS were used as the reference and the range of the samples was 11.0 to $427.5 \mathrm{nmol} / \mathrm{L}$ as measured by LCMS/MS. Values $<20.0 \mathrm{nmol} / \mathrm{L}$ were excluded from statistical analyses using the Architect method, as its lower measurement range is $20.0 \mathrm{nmol} / \mathrm{L}$ according to the manufacturer. Values $>175.0 \mathrm{nmol} / \mathrm{L}$ were excluded from statistical analyses with the Cobas method, as its upper measurement range is 175.0 $\mathrm{nmol} / \mathrm{L}$ according to the manufacturer. Overall, 83 complete cases of the 100 examined could be evaluated for Architect and, 79 complete cases could be evaluated for Cobas statistical analysis. $25(\mathrm{OH}) \mathrm{D}_{2}$ concentrations were undetectable in $\mathrm{LC} /$ MS-MS measurements. Thus, all comparison data were obtained from Architect i2000, Cobas e601, and LC-MS/MS represented $25(\mathrm{OH}) \mathrm{D}_{3}$ solely. We found that all data from the three different assay methods were not distributed normally based on Kolmogorov-Smirnov tests $(P<0.001)$. The examined ranges of the $25(\mathrm{OH}) \mathrm{D}$ concentrations (minimum to maximum) were $9.1-352.5 \mathrm{nmol} / \mathrm{L}$ as measured by LCMS/MS, 7.5-129.0 nmol/L as measured by Cobas e601, 26.5- $96.5 \mathrm{nmol} / \mathrm{L}$ as measured by Architect i2000.

The slope and intercept of the Passing-Bablok regression line for each immunoassay method were compared with those of the LC-MS/MS assay meth- 
od. Details regarding the Passing-Bablok regression analysis are shown in Table 1.

Differences and biases between methods were evaluated using a Bland-Altman plot (Figure 1 and 2).
$25(\mathrm{OH}) \mathrm{D}$ results were classified to define vitamin $D$ status, as recommended by The Endocrine Society's Clinical Practice Guideline on Vitamin D (20). We compared the proportion of samples fulfilling vitamin $D$ deficiency using the $50 \mathrm{nmol} / \mathrm{L}$ cut-off by different assays using $\mathrm{K}$ analysis (Table 2 ).

TABLE 1. Details regarding the Passing-Bablok regression analysis.

\begin{tabular}{ccc}
\hline Statistical analysis & $\begin{array}{c}\text { LC-MS/MS (variable x) } \\
\text { Architect i2000 (variable y) } \\
\text { (nmol/L) }\end{array}$ & $\begin{array}{c}\text { LC-MS/MS (variable x) } \\
\text { Cobas e601 (variable y) } \\
\text { (nmol/L) }\end{array}$ \\
\hline $\mathbf{N}$ & 83 & 79 \\
\hline $\begin{array}{c}\text { Regression equation } \\
\text { Random differences }\end{array}$ & $\mathrm{y}=17.08(12.98-21.39)+0.77(0.67-0.85) \mathrm{x}$ & $\mathrm{y}=-5.23(-8.73-0.19)+0.97(0.77-1.15) \times$ \\
\hline 1.96 RSD Interval & 23.35 & 12.25 \\
\hline $\begin{array}{c}\text { Linear model validity } \\
\text { Cusum test for linearity }\end{array}$ & -45.76 to 45.76 & -24.01 to 24.01 \\
\hline
\end{tabular}

RSD - residual standard deviation, $\mathrm{Cl}$ - confidence interval.

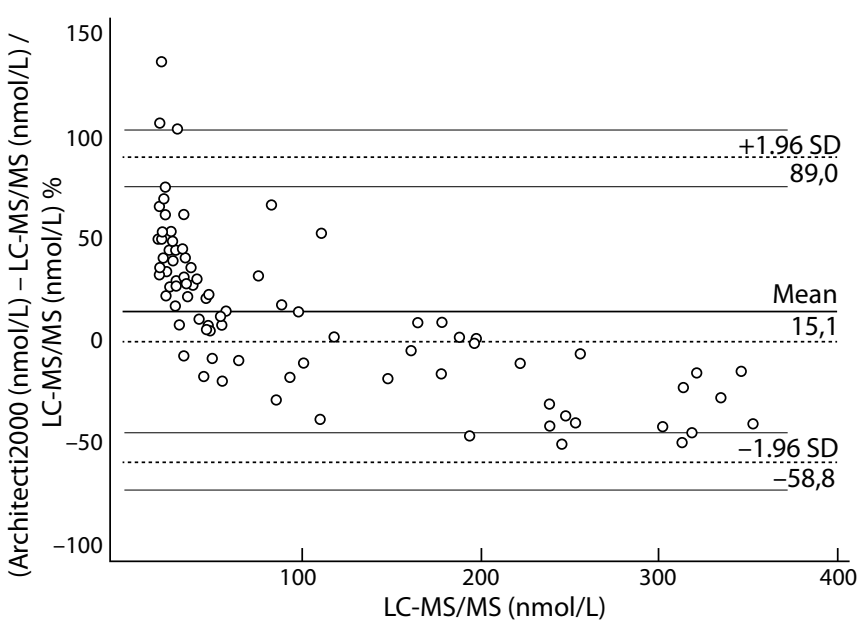

Figure 1. Comparison of the Abbott Architect i2000 25-OH Vitamin D immunoassay method with LC-MS/MS method using Bland-Altman analysis.

\section{Discussion}

Our results revealed that both immunoassays demonstrated acceptable performance. The results of both methods were comparable to those of LC-MS/MS based on Passing Bablok regression

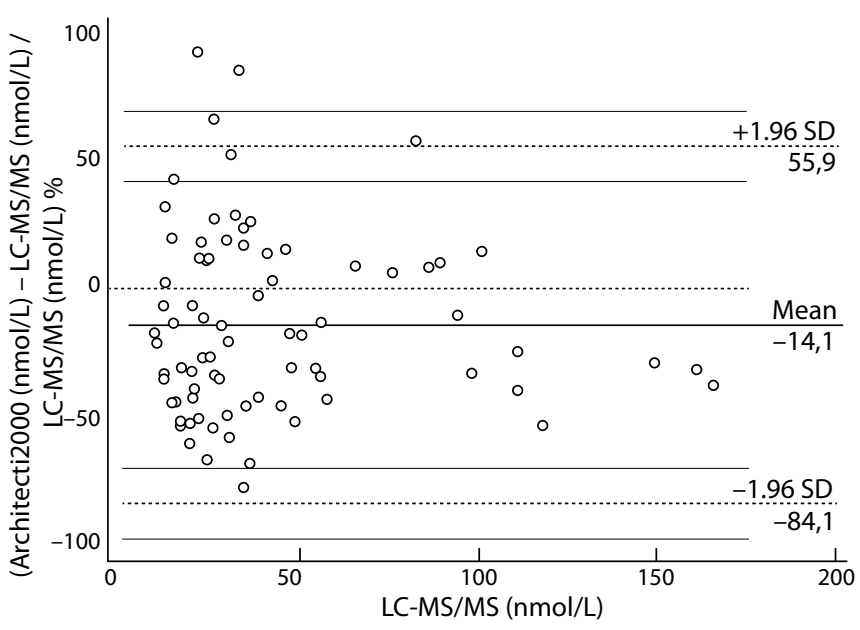

Figure 2. Comparison of the Roche Cobas e601 Vitamin D total immunoassay method with LC-MS/MS method using BlandAltman analysis.

analysis. A mean bias of $15.1 \%$ was observed for the Abbott Architect and a mean bias of $-14.1 \%$ was observed for the Roche assay based on the Bland-Altman difference plots. These biases may 
TABLE 2. Classification of vitamin D status and inter-rater agreement between LC-MS/MS and two immunoassay methods according to $25(\mathrm{OH}) \mathrm{D}$ based on $50.0 \mathrm{nmol} / \mathrm{L}$ cut-off.

\begin{tabular}{|c|c|c|c|c|}
\hline$N=83$ & $\begin{array}{c}\text { Severe deficiency } \\
\leq \mathbf{2 5 . 0} \\
\mathrm{nmol} / \mathrm{L}\end{array}$ & $\begin{array}{c}\text { Deficiency } \\
26.0-50.0 \\
\mathrm{nmol} / \mathrm{L}\end{array}$ & $\begin{array}{c}\text { Insufficiency } \\
51.0-74.0 \\
\mathrm{nmol} / \mathrm{L} \\
\end{array}$ & $\begin{array}{c}\text { Sufficiency } \\
\geq 75.0 \\
\mathrm{nmol} / \mathrm{L} \\
\end{array}$ \\
\hline Architect i2000 & 0 & 37 (44\%) & 14 (17\%) & 32 (39\%) \\
\hline LC-MS/MS & 15 (18\%) & 29 (35\%) & $5(6 \%)$ & $34(41 \%)$ \\
\hline \multicolumn{5}{|l|}{$N=79$} \\
\hline Cobas e601 & $40(50 \%)$ & $23(29 \%)$ & $6(8 \%)$ & 10 (13\%) \\
\hline LC-MS/MS & $32(40 \%)$ & $29(37 \%)$ & $5(7 \%)$ & $13(16 \%)$ \\
\hline \multirow[t]{2}{*}{ LC-MS/MS } & \multicolumn{2}{|c|}{$\begin{array}{c}\mathrm{N}=\mathbf{8 3} \\
\text { Architect i2000 }\end{array}$} & \multicolumn{2}{|c|}{$\begin{array}{c}N=79 \\
\text { Cobas e601 }\end{array}$} \\
\hline & \multicolumn{2}{|c|}{$\begin{array}{c}\text { Architect i2000 } \\
<50.0>50.0 \\
(\mathrm{nmol} / \mathrm{L})\end{array}$} & \multicolumn{2}{|c|}{$<50.0>50.0$} \\
\hline$<50.0 \mathrm{nmol} / \mathrm{L}$ & \multicolumn{2}{|l|}{37} & \multicolumn{2}{|c|}{61} \\
\hline$>50.0 \mathrm{nmol} / \mathrm{L}$ & \multicolumn{2}{|c|}{39} & 2 & 16 \\
\hline к $(95 \% \mathrm{Cl})$ & \multicolumn{2}{|c|}{$0.83(0.71-0.95)$} & \multicolumn{2}{|c|}{$0.93(0.82-1.03)$} \\
\hline
\end{tabular}

$\mathrm{Cl}$ - confidence interval, $\mathrm{K}$ - Kappa.

25(OH)D results were classified to define vitamin D status, as recommended by The Endocrine Society's Clinical Practice Guideline on Vitamin D (20).

lead to overestimation or underestimation of 25(OH)D concentrations. In addition, both methods had some limitations when their performance was challenged with samples containing low and high total 25(OH)D concentrations. We found higher concentration-dependent deviation for using Abbott Architect measurements compared to that using LC-MS/MS. The Roche assay showed better performance than the Abbott assay.

Consistent with the findings of this study, Farrell et al. found a mean bias of $40.9 \%$ between the Abbott Architect and LC-MS/MS methods for whole study samples ( $\mathrm{N}=170$ ) with concentrations ranging from 5.0 to $151.0 \mathrm{nmol} / \mathrm{L}$ and a mean bias of $104.5 \%$ for samples with concentrations $<20.0$ $\mathrm{nmol} / \mathrm{L}$ as assessed by LC-MS/MS (22). They suggest that this excessive positive bias does not present a serious problem when the assay is used according to the manufacturer's specification, which recommends not reporting results $<20.0 \mathrm{nmol} / \mathrm{L}$ (21). In our study, although the samples with concentrations $<20.0 \mathrm{nmol} / \mathrm{L}$ were excluded from analysis, a mean bias of $15.1 \%$ was found. Holmes et al. found that the Abbott Architect assay had a bias of $40 \%$ at $25(\mathrm{OH}) \mathrm{D}_{3}$ concentrations $<50.0$ $\mathrm{nmol} / \mathrm{L}(\mathrm{N}=94)$, and they suggest that this positive bias for samples at the lower end of the analytical measuring range is likely due to a standardization or calibration defect or a common, positive interfering substance that was not measured by LC-MS/MS (22). Ong et al. also found significant positive bias for the Abbott Architect assay compared to LC-MS/MS ( $\mathrm{N}=200)(23)$. Clinically, $25(\mathrm{OH})$ D concentrations $<50.0 \mathrm{nmol} / \mathrm{L}$ indicate vitamin $\mathrm{D}$ deficiency; thus, inaccuracies at low concentrations have a limited impact on treatment decisions. In this study, the Roche Cobas Vitamin D total assay method showed a bias of $-14.1 \%$. Previous studies evaluating the Cobas methods found negative bias compared to that of LC-MS/MS, which was similar to the results of our study $(24,25)$.

There are several explanations for the observed inter-method differences between the immunoassays and LC-MS/MS. First, the differences between the immunoassays and LC-MS/MS method may have resulted from different calibrator traceability. The Roche Cobas Vitamin D total assay has been standardized against LC-MS/MS which in turn has been standardized to the NIST standard. The Abbott Architect 25-OH Vitamin D assay is traceable 
to the manufacturer's internal standard (primary calibrator) against an absorbance of $264 \mathrm{~nm}$. This may explain the better agreement between the Roche method and LC-MS/MS compared to the Abbott method. Additionally, cross-reactivity may have occurred with other metabolites of 25(OH)D. All immunoassays for $25(\mathrm{OH}) \mathrm{D}$ show high cross-reactivity with $24,25(\mathrm{OH})_{2} \mathrm{D}$, which may be present in the serum at concentrations of up to about 10-15 $\mathrm{nmol} / \mathrm{L}$ (12). The presence of the $24,25(\mathrm{OH})_{2} \mathrm{D}$ can lead to increased 25(OH)D concentrations measured by immunoassays. The Abbott assay manufacturer states that $112 \%$ cross-reactivity occurs with $24,25(\mathrm{OH})_{2} \mathrm{D}_{3}$, while Roche assay manufacturer claims to have $149 \%$ cross-reactivity with $24,25(\mathrm{OH})_{2} \mathrm{D}_{3}$. In addition, differential cross-reactivity of $25(\mathrm{OH}) \mathrm{D}_{2}$ in immunoassays is another potential problem; however, this was not an issue for the differences observed between the assays in our study because $25(\mathrm{OH}) \mathrm{D}_{2}$ concentrations were undetectable in the studied samples. This situation may be explained by vitamin $D_{3}$ supplementation which is common in our region.

Matrix effects are known to occur in immunoassays and can lead to false high or low results. The most important type of matrix effect is any effect that occurs between the matrix in the calibrants and the patient samples (13). Abbott assay's calibrators are composed of phosphate-buffered saline containing heat-inactivated horse serum and Roche assay's calibrators contain human serum as a matrix. Another factor may be the ability of an assay to separate $25(\mathrm{OH}) \mathrm{D}$ from its binding protein. In LC/MS-MS methods, $25(\mathrm{OH}) \mathrm{D}$ is separated from its binding protein by solvent extraction. However, in immunoassay methods, solvent extraction and chromatographic separation have been replaced by various blocking agents that displace $25(\mathrm{OHD})$ from VDBP, which shows varying success. Although this simplified sample pre-treatment method enables the use of high samplethroughput and automation, in case of incomplete extraction, false low 25(OH)D concentrations may be obtained. Strong binding between the highly hydrophobic 25(OH)D and VDBP creates competition with the capturing antibodies. VDBP must be be inactivated or completely removed from the sample, as residual active VDBP at concentrations as low $4 \mathrm{nmol} / \mathrm{L}(0.5 \%$ of total VDBP) may interfere with the assay $(10,11,26)$. A recent study, including 50 healthy individuals, 52 pregnant women, 50 hemodialysis patients, and 50 intensive care patients suggested that in automated assays, not all of the 25(OH)D was extracted from the VDBP (27). The authors observed an inverse relationship between VDBP concentrations and deviations of immunoassay results from LC-MS/MS results. The Abbott assay (Architect i2000) showed VDBP concentration-dependent differences, but the Roche assay (Modular Analytics E170) did not show these differences (27). The Abbott assay uses 8-anilino-1 naphthalenesulfonic acid in triethanolamine methanol buffer to separate 25(OH)D from VDBP. In the Roche assay, pre-treatment of the sample involves an acidic $\mathrm{pH}$ change (with dithiothreitol) which inactivates the DBP and separates the bound $25(\mathrm{OH})$ D.

The Endocrine Society's Clinical Practice Guideline on Vitamin $D$ suggests that $25(\mathrm{OH}) \mathrm{D}$ levels below $50.0 \mathrm{nmol} / \mathrm{L}$ are deficient, $51.0-75.0 \mathrm{nmol} / \mathrm{L}$ are insufficient, and 75.0-250.0 nmol/L are sufficient (20). After classification of the $25(\mathrm{OH}) \mathrm{D}$ results according to recommendations of The Endocrine Society, we analyzed inter-rater agreement using the $\mathrm{K}$ statistic and found strong to nearly perfect agreement in vitamin D status between the immunoassays and LC-MS/MS. These results are consistent with the findings of previous studies $(22,28)$.

This study had some limitations. According to CLSI EP09-A3 specifications, analysis by comparative and test methods should be performed within a time span consistent with analyte stability. For all analytes, the time span until analysis should not exceed $2 \mathrm{~h}$ for analysis for each method and, if possible, samples should be drawn on the day of analysis. In this study, because the $25(\mathrm{OH}) \mathrm{D}$ measurements by LC-MS/MS were performed in a different hospital, measurements by immunoassay and LC-MS/MS were performed within different time spans. Additionally, we did not investigate the influence of C3-epi-25(OH)D. The LC-MS/MS method used in this study could not separate the different epimer forms of vitamin $D$, particularly 3-epi-25(OH) $\mathrm{D}_{3}$ and 3-epi-25(OH) $\mathrm{D}_{2}$. If these 
epimers are available in samples, assay methods using 25(OH)D measurements may detect epimers within total $25(\mathrm{OH}) \mathrm{D}$ concentrations without separation, leading to biases due to cross-reactions. $A$ recent study by Farrell et al. detected C3-epi$25(\mathrm{OH}) \mathrm{D}_{3}$ in $40.4 \%$ of healthy adults; the authors suggested that the C3-epimer of $25(\mathrm{OH}) \mathrm{D}$ was a source of analytical variance in immunoassays (29). Another limitation may be the difference in treatment between the samples used in the immunoassays (fresh) and the LC-MS/MS (one freeze/thaw cycle), but a recent study reported that long-term frozen storage does not affect serum vitamin $D$ levels and that $25(\mathrm{OH}) \mathrm{D}$ is stable for 7 days at -20 ${ }^{\circ} \mathrm{C}$ (30). Additionally, sample collection tube used in this study may be possible preanalytical source of differences on different assays. However, in a previous study, the difference between serum separator tube with clot activator and EDTA tube was investigated and there was no difference between serum and plasma vitamin D concentration; the authors suggested that the choice of collection tube was not to affect vitamin D concentration (30).

\section{Conclusion}

In this study, we found that both immunoassays demonstrated acceptable performance, but had some limitations when their performance was challenged with samples containing low and high total $25(\mathrm{OH}) \mathrm{D}$ concentrations. We found that the deviation increased in a concentration-dependent manner using Abbott Architect measurements compared to using LC-MS/MS. The Roche Cobas assay demonstrated better performance than the Abbott Architect in the studied samples. Therefore, immunoassay methods can give variable results, which is most apparent when the immunoassays are used to evaluate with a range of samples that challenge their analytical performance. Laboratory professionals should be aware of these issues when changing methods in their routine work and comparing results obtained from different platforms.

\section{Acknowledgments}

The authors are thankful to Roche Diagnostics and Abbott Diagnostics for providing reagents for this study.

\section{Potential conflict of interest}

Roche Diagnostics and Abbott Diagnostics provided reagents for this study.

5. Freedman DM, Looker AC, Chang SC, Graubard BI. Prospective study of serum vitamin $D$ and cancer mortality in the United States. J Natl Cancer Inst 2007;99:1594-602. http:// dx.doi.org/10.1093/jnci/djm204.

6. Holick MF. Vitamin D status: measurement, interpretation and clinical application. Ann Epidemiol 2009;19:73-8. http://dx.doi.org/10.1016/j.annepidem.2007.12.001.

7. Lai JK, Lucas RM, Clements MS, Harrison SL, Banks E. Assessing vitamin D status: pitfalls for the unwary. Mol Nutr Food Res 2010;54:1062-71. http://dx.doi.org/10.1002/ mnfr.200900468.

8. Hollis BW. Measuring 25-hydroxyvitamin D in a clinical environment: challenges and needs. Am J Clin Nutr 2008;88:507S-10S.

4. Vanlint S. Vitamin D and obesity. Nutrients 2013;5:949-56. http://dx.doi.org/10.3390/nu5030949. 
9. Tai SS, Bedner M, Phinney KW. Development of a candidate reference measurement procedure for the determination of 25-hydroxyvitamin D3 and 25-hydroxyvitamin D2 in human serum using isotope-dilution liquid chromatographytandem mass spectrometry. Anal Chem 2010;82:1942-8. http://dx.doi.org/10.1021/ac9026862.

10. Fraser WD, Milan AM. Vitamin D assays: past and present debates, difficulties, and developments. Calcif Tissue Int 2013;92:118-27. http://dx.doi.org/10.1007/s00223-0129693-3.

11. Farrell CJ, Herrmann M. Determination of vitamin $D$ and its metabolites. Best Pract Res Clin Endocrinol Metab 2013;27:675-88. http://dx.doi.org/10.1016/j. beem.2013.06.001.

12. Carter GD. 25-hydroxyvitamin $D:$ a difficult analyte. Clin Chem 2012;58:486-8. http://dx.doi.org/10.1373/ clinchem.2011.180562.

13. Wallace $A M$, Gibson $S$, de la Hunty $A$, Lamberg-Allardt $C$, Ashwell M. Measurement of 25-hydroxyvitamin D in the clinical laboratory: current procedures, performance characteristics and limitations. Steroids 2010;75:477-88. http:// dx.doi.org/10.1016/j.steroids.2010.02.012.

14. Thienpont LM, Stepman HC, Vesper HW. Standardization of measurements of 25-hydroxyvitamin D3 and D2. Scand J Clin Lab Invest Suppl. 2012;243:41-9.

15. CLSI. Measurement procedure comparison and bias estimation using patient samples; approved guideline - third edition. CLSI document EP09-A3, Wayne, PA, USA, Clinical and Laboratory Standards Institute, 2013.

16. Sahillioglu B, Serdar MA, Erkal N, Erden G, Bakir F, Yildirimkaya MM, et al. Method validation of tandem mass spectrometry for 25-Hydroxyvitamin D3 and comparison of this method with other methods. Turk J Biochem 2011;36:73-9.

17. Bilić-Zulle L. Comparison of methods: Passing and Bablok regression. Biochem Med (Zagreb) 2011;21:49-52. http:// dx.doi.org/10.11613/BM.2011.010.

18. Bland JM, Altman DG. Comparing methods of measurement-why plotting difference against standard method is misleading. The Lancet 1995; 346:1085-7. http://dx.doi. org/10.1016/S0140-6736(95)91748-9.

19. McHugh ML. Interrater reliability: the kappa statistic. Biochem Med (Zagreb) 2012;22:276-82. http://dx.doi. org/10.11613/BM.2012.031.

20. Holick MF, Binkley NC, Bischoff-Ferrori HA, Gordon CM, Hanley $D A$, Heaney $R P$, et al. Guidelines for preventing and treating vitamin $D$ deficiency and insufficiency revisited. J Clin Endocrinol Metab 2012;97:1153-8. http://dx.doi. org/10.1210/jc.2011-2601.
21. Farrell CJ, Martin S, McWhinney B, Straub I, Williams $P_{,}$ Herrmann M. State of-the-art vitamin $D$ assays: a comparison of automated immunoassays with liquid chromatography-tandem mass spectrometry methods. Clin Chem 2012;58:531-42. http://dx.doi.org/10.1373/ clinchem.2011.172155.

22. Holmes EW, Garbincius J, McKenna KM. Analytical variability among methods for the measurement of 25-hydroxyvitamin D: still adding to the noise. Am J Clin Pathol 2013;140:550-60. http://dx.doi.org/10.1309/AJCPU2SKW1TFKSWY.

23. Ong L, Saw S, Sahabdeen NB, Tey KT, Ho CS, Sethi SK. Current 25-hydroxyvitamin $D$ assays: do they pass the test? Clin Chim Acta 2012;413:1127-34. http://dx.doi. org/10.1016/j.cca.2012.03.009.

24. Denimal D, Ducros V, Dupré T, Dousset B, Meunier C, Aho $S$, et al. Agreement of seven 25-hydroxy vitamin $D_{3}$ immunoassays and three high performance liquid chromatography methods with liquid chromatography tandem mass spectrometry. Clin Chem Lab Med 2014;52:511-20. http://dx.doi.org/10.1515/cclm-2013-0434.

25. Wielders JP, Carter GF, Eberl H, Morris G, Jürgen Roth $H$, Vogl C. Automated Competitive Protein-Binding Assay for Total 25-OH Vitamin D, Multicenter Evaluation and Practical Performance. J Clin Lab Anal 2014; Aug 17. doi: 10.1002/jcla.21793. [Epub ahead of print]. http://dx.doi. org/10.1002/jcla.21793.

26. Depreter B, Heijboer AC, Langlois MR. Accuracy of three automated 25-hydroxyvitamin $D$ assays in hemodialysis patients. Clin Chim Acta 2013;415:255-60. http://dx.doi. org/10.1016/j.cca.2012.10.056.

27. Heijboer AC, Blankenstein MA, Kema IP, Buijs MM. Accuracy of 6 routine 25-hydroxyvitamin $D$ assays: influence of vitamin $D$ binding protein concentration. Clin Chem 2012;58:543-8. http://dx.doi.org/10.1373/ clinchem.2011.176545.

28. Nguyen VT, Li X, Castellanos KJ, Fantuzzi G, Mazzone T, Braunschweig CA. The accuracy of vitamin $D$ assays of circulating 25-hydroxyvitamin D values: influence of 25-hydroxyla ted ergocalciferol concentration. J AOAC Int 2014;97:104855. $h$ ttp://dx.doi.org/10.5740/jaoacint.13-305.

29. Farrell C, Soldo J, Williams P, Herrmann M. 25-Hydroxyvitamin $D$ testing: challenging the performance of current automated immunoassays. Clin Chem Lab Med 2012;50:195363. $\mathrm{http} / / / \mathrm{dx}$.doi.org/10.1515/cclm-2012-0522.

30. Colak A, Toprak B, Dogan N, Ustuner F. Effect of sample type, centrifugation and storage conditions on vitamin D concentration. Biochem Med (Zagreb) 2013;23:321-5. http://dx.doi.org/10.11613/BM.2013.039. 